\title{
LA DERROTA DEL MARKETING: ¿REALIDAD O NUEVOS DESAFÍOS GERENCIALES?*
}

\author{
Etienne Cracco \\ CEnTRo de Estudios En Gestión INTERnACIONAL \\ ICEP, BÉLGICA
}

\section{Resumen}

Como resultado de una investigación propia, el expositor sistematiza los errores que usualmente cometen las empresas al aplicar nuevas estrategias de marketing. Describe trece grandes errores a partir de la experiencia real de prestigiosas empresas de Europa y Estados Unidos, explica las razones de los fracasos y establece las lecciones que se puede extraer de ellos. Las fuentes de errores se descubren en el inadecuado manejo del análisis del mercado, la cultura empresarial y organizacional, los sistemas de información, el factor tecnológico y los canales de comunicación.

Q uizá una de las cosas más interesantes de analizar sean los fracasos, porque a veces se aprende mucho más de los problemas y de los fracasos que de los éxitos. Analizar los fracasos proporciona un sentido de humildad a la docencia; por ello, como profesor de marketing, voy a centrar mi exposición en este tema.

* Conferencia dictada en ESAN el 14 de julio de 2001 como parte de los actos de celebración del primer «Día del Graduado». Transcrita con autorización del expositor y adaptada de la versión oral por Ada Ampuero.
En los últimos treinta años, el marketing ha sido plenamente aceptado y se ha convertido en uno de los aspectos sustanciales de las empresas. Las inversiones en marketing han sido tan grandes en comparación con las realizadas en otras áreas o funciones de la empresa que se podría pensar que en el marketing ya todo está resuelto. Sin embargo, cuando uno habla con gerentes generales parece que esto no es así, que el marketing ha sido insuficiente y aun contraproducente para dirigir las empresas hacia sus metas. De ahí el título de la conferencia: ¿es la de- 
rrota del marketing una realidad o hay detrás nuevos desafíos gerenciales? Esto es lo que vamos a analizar a continuación.

A partir de la década de los ochenta, las inversiones en marketing crecieron, en general, $10 \%$ más rápido que las otras inversiones, pero en los cinco o siete últimos años hubo un cambio y ese ritmo de crecimiento fue disminuyendo. Ésta es la razón del concepto de la derrota del marketing, porque en términos relativos se dejó de invertir o se desarrolló una estabilidad relativa dentro de los presupuestos de marketing. Durante ese mismo periodo, la tasa de fracasos del lanzamiento de nuevos productos o de la consolidación de la participación de productos y de empresas en el mercado se ha mantenido igual. Después de tanta inversión, no hubo reducción de número de especialistas de marketing ni se puede notar un mejoramiento en este campo, lo que es muy preocupante. ¿Por qué razón se invierte mucho más en el campo del marketing y no se observa resultados alentadores? La tasa de fracasos, por ejemplo, sigue siendo la misma. Se sabe que sólo uno de cada diez productos será exitoso, proporción que es muy baja.

He realizado una investigación sobre múltiples casos de empresas prestigiosas que experimentaron rotundos fracasos luego de realizar elevadas inversiones en marketing. Mis objetivos han sido, en primer lugar, determinar cuáles fueron las grandes razones de estos fracasos, es decir, identificar las fuentes de errores, $y$, en segundo lugar, establecer qué lecciones se puede sacar de esos errores. De esta manera, puedo decirles que hay trece grandes errores que usualmente cometen las empresas que fracasan. Voy a expli- car en qué consiste cada uno de éstos refiriéndome a ejemplos concretos.

Uno de los grandes fracasos de los últimos años es el de Monsanto, empresa que hasta hace dos años era la líder en biotecnología y hoy ya no existe. Cuando estaba yéndose a la quiebra fue comprada por una empresa sueca, la que ahora está tratando infructuosamente de vender el área agrícola de Monsanto. Otro caso muy interesante de fracaso es el de Fruitopia, una especie de gaseosa de la Coca Cola en la cual se invirtió millones y millones de dólares hace solamente tres años. También podemos mencionar aquí el caso del Wap, lo mejor que podía haber para telefonía celular y que, sin embargo, fue enterrado al cabo de un año y luego de la pérdida de más de mil millones de dólares invertidos. Otro caso que ha sido extraordinario como fracaso de marketing es el de Milenium Dome, el elefante blanco de Tony Blair, quien ha impulsado la construcción arquitectónica en Londres y quiso hacer de éste el máximo proyecto del siglo XXI. Incluso la reina Isabel II tuvo que participar con Tony Blair el 31 de diciembre en el gran espectáculo que rodeó su apertura. Hoy el Milenium Dome está cerrado y quebrado, con un considerable monto de inversión adentro. Otro caso considerado también un fracaso de marketing es el de la cerveza Loburg.

Los casos anteriores corresponden a productos o empresas, pero los fracasos también envuelven a marcas o nombres muy bien conocidos. Philips, por ejemplo, que ha abandonado sus electrodomésticos a Whirlpool; o Motorola, conocida por sus radios, que ha cambiado todos sus rubros y cuyos productos de hace veinte años ya casi no existen en el mercado; o Polaroid, la empresa de fotogra- 
fía instantánea, que está a punto de quebrar.

En el caso de las nuevas tecnologías, los casos de reveses empresariales son aun más interesantes. Un caso de gran magnitud es el de Quantum.com, empresa que ha quebrado. Otro caso notable es el de Ready.be, empresa que quería ingresar en el negocio de la distribución y ha quebrado después de siete meses de existencia y tres millones y medio de dólares de inversión. El caso más saltante, sin embargo, es el de Leernhout \& Hauspie, empresa belga que era líder mundial en software de reconocimiento de voz y que hoy ha hundido no solamente el capital de sus accionistas, sino el de toda una región que había invertido en este campo.

He mencionado todos estos ejemplos por una razón muy sencilla, para demostrar que los fracasos existen; yo podría sacar un libro entero de fracasos. ¿Cuáles fueron las razones de estos fracasos, qué errores se cometieron y qué o quiénes fueron los responsables? ¿El concepto de marketing, la incapacidad o ignorancia de los gerentes de marketing, los estrategas que no pudieron informar o comunicar sus planes al resto de la empresa, el entorno que cambia demasiado rápidamente o la dinámica organizacional interna de las empresas que no puede ajustarse a los cambios y al entorno competitivo globalizado?

El primer error es un error bastante clásico: «Yo soy el líder del mercado y quién me va a sacar». Éste es el momento más peligroso, porque cuando se es el líder y no se toma conciencia de lo que puede ocurrir, el único camino que se puede recorrer es el de la caída. El caso más espectacular ocurrido hasta hoy es el de Polaroid, líder en la fotografía instantánea. Durante años se decía que la foto Polaroid no valía mucho, pero se vendían cantidades considerables de películas Polaroid y ni Kodak ni Fuji podían competir con esta empresa en este segmento del mercado. Sobre la base de estos elementos, los gerentes de Polaroid no prestaron gran atención a la fotografía digital. Pensaron que fracasaría, que seguirían siendo los únicos, que la foto instantánea era la más atractiva porque la gente preferiría ver su foto revelada y fijada en papel en 10 ó 20 segundos. Hoy nadie quiere usar una Polaroid; se prefiere la cámara digital, que permite ver de inmediato aquello que se fotografía. ¿A qué puede atribuirse la responsabilidad de este error? A la prepotencia de la empresa, a la falta de visión estratégica de largo plazo y también a un error de marketing. A los gerentes de Polaroid no se les ocurrió pensar que en cualquier momento otra empresa podría sacarlos del mercado. Otro caso típico de este error fue el de IBM, que en un momento dado pensó que ninguna otra empresa podía «tocarla» en el campo de la informática; hoy se ha vuelto más sabia en este aspecto.

El error número dos consiste en suponer que: «una revolución tecnológica será adoptada rápidamente». Cuando se produce una revolución tecnológica que implica innovaciones y beneficios obvios, ¿qué se hace si el mercado no los toma de inmediato, qué se hace si todavía hay gente que prefiere usar escobas a pesar de toda la tecnología disponible? Dos casos muy interesantes al respecto son el de Wap y el de Leernhout \& Hauspie.

Wap fue resultado de un análisis de mercado que estableció que tanto la In- 
ternet como los teléfonos celulares son utilizados mayormente por el mercado de jóvenes y que, por lo tanto, había necesidad de integrar los dos servicios. Nokia, la empresa que impulsó el desarrollo de esta tecnología, invirtió millones de dólares en la integración de la Internet y el celular; sin embargo, lo que se logró fue un celular muy lento y costoso que se convirtió en un fracaso total. El caso del Wap impactó fuertemente en la bolsa, ya que las pérdidas, al tener que absorberse, causan un impacto negativo sobre los resultados.

¿Qué sucedió? Primero se tiene que analizar el costo de la tecnología, no solamente en términos de dinero, sino en tiempo. ¿Cuánto tiempo se necesita para aprender o cuánto tiempo se dedica a estas herramientas tecnológicas? Los jóvenes de hoy no quieren esperar 10 minutos para ingresar a la Internet, quieren hacerlo en 5 segundos; de otro modo no les sirve. Además, el costo de esta revolución tecnológica era demasiado alto en comparación con los beneficios que produciría. ¿El resultado?: un desastre. ¿A qué puede atribuirse este error número dos? A la falta de análisis de la dinámica del mercado y de la dimensión humana, y también a la orientación tecnológica de la producción de la empresa, que ocurre cuando los ingenieros «se enamoran» de su tecnología. Esta orientación está presente en la mayoría de las empresas de nueva tecnología, y uno tiene que volverse crítico de estos aspectos.

El error número tres es el síndrome competitivo. Muchos autores se refieren a la competencia en términos bélicos. En efecto, en este caso la empresa tiende a armarse con las mismas armas de la competencia sin detenerse a pensar si real- mente las necesita o si le serán útiles. Si el rival lanza un nuevo producto, la empresa necesita también ese producto. Se supone que si el competidor lo hace es porque lo debe haber reflexionado. Lo exitoso del competidor debe ser imitado para evitar quedar distanciado. El caso típico que puede servir para ilustrar este error es el de la Coca Cola. Su principal competidora, la Pepsi Cola, obtuvo un gran éxito en un nicho del mercado con un nuevo producto que no era ni gaseosa ni jugo natural, sino una mezcla de jugo natural, gaseosa y producto energético. ¿Qué hizo entonces la Coca Cola? Para evitar quedar rezagada lanzó un producto similar sin analizar si éste era o no conveniente para ella. ¿El resultado?: un desastre total. La Coca Cola ha perdido imagen entre los canales de distribución, en la profesión y en la bolsa. ¿Qué causas están detrás de este error? La falta de visión estratégica; debió analizarse si el producto se ajustaba a la cultura y a la visión estratégica de la empresa. Lo que está ocurriendo en muchos casos es que la empresa ingresa a un mercado nicho cuando el competidor está en un mercado de gran consumo masivo.

Les voy a dar otro ejemplo interesante, el de una empresa belga productora de los más famosos chocolates de ese país: Cote d'Or. En una ocasión una empresa suiza sacó un bombón de tipo natural y entonces la empresa belga decidió que tenía que lanzar un producto similar $-\mathrm{y}$ como tenía que hacerlo rápido le puso a la golosina el nombre de la chica encargada de los baños, sólo una pequeña historia para mostrar cómo surgen las marcas de marketing-. ¿Qué ocurrió con Cote d'Or? Fue comprada por la empresa suiza, aunque no por esta razón. Dentro de un conjunto competitivo, la experiencia 
descrita muestra falta de creatividad, una estrategia competitiva reactiva, una gerencia que reacciona sólo cuando hay crisis, y falta de confianza en la propia creatividad. En Cote d'Or había profesionales que sabían lo qué se necesitaba y presentaban ideas innovadoras antes que la competencia, pero no se les daba crédito. Por lo tanto, hubo también fallas de cultura empresarial y de comunicación interna.

El error número cuatro es la tecnoinvesticracia del departamento de marketing: cuando la manipulación de datos llega a dominar la creatividad gerencial. Esto es muy peligroso y en parte se origina en las escuelas de negocios, porque se le da tanta importancia al análisis de los datos que se forma profesionales que confían solamente en los datos y no en el sentido común de la gente. Uno debe utilizar los datos para sustentar o confirmar el sentido común, no a la inversa. Para ilustrar este error voy a tomar el caso de la cerveza Loburg, de la empresa cervecera Interbrew, que produce 200 marcas de cervezas distintas para cubrir el mercado y en la actualidad ocupa el segundo o tercer lugar en el mundo. ¿Qué sucedió? Manipulando todo los datos del mercado, un especialista de marketing identificó que las mujeres jóvenes, entre 18 y 29 años de edad, no tomaban cerveza, y que el segmento de estrato medio alto, intelectual y un poco elitista tampoco lo hacía. También encontró que algunas mujeres jóvenes empezaban a consumir una cerveza danesa llamada Carlsberg. En consecuencia, Interbrew lanzó la cerveza Loburg.

Después de 10 años de inversión permanente, de ilusión permanente, se llegó a la conclusión de que las chicas entre 18 y 29 años no toman cerveza porque este producto hace engordar, mientras que los intelectuales toman, a lo más, bebidas tipo gin o coctéles, pero no cerveza. ¿El resultado?: un desastre total. Finalmente, Interbrew consiguió la distribución de Carlsberg. La responsabilidad por este error recae en la cultura empresarial, la dimensión productora-tecnócrata de los servicios de marketing y la incapacidad de asumir la incertidumbre. Cuídense de que los hombres de marketing les propongan investigación tras investigación. Uno debe tomar distancia del componente afectivo y volverse crítico, analítico y positivo al mismo tiempo. Aceptar los propios errores es algo muy importante.

El siguiente error, el número cinco, ocurre cuando el poder económico y tecnológico domina al político y no se le da importancia al entorno social. Éste es uno de los errores de mayor actualidad; es el caso de Monsanto y también el de Midland.

Monsanto ilustra el caso de cómo del éxito se puede llegar al fracaso total. Monsanto desarrolló organismos genéticamente modificados y consiguió la autorización de la FDA y del Ministerio de Agricultura de Estados Unidos para comercializarlos. Los agricultores vieron los certificados, las garantías, aceptaron el producto y obtuvieron un gran impacto: un incremento de $20 \%$ y hasta de $30 \%$ en la cosecha. El producto era realmente bueno y logró una difusión y una penetración de mercado excepcional.

Entonces, primer error, el presidente de la empresa se dio cuenta de que si el agricultor podía obtener semillas de la cosecha, no compraría semillas todos los años. Con el fin de evitar esto, dio ins- 
trucciones a su departamento de investigación y desarrollo para que introdujera en la semilla una pequeña bacteria o enzima que causara su autodestrucción. El éxito fue total, los agricultores compraban semillas todos los años y Monsanto se convirtió en la estrella de la biotecnología. Con estos resultados, la empresa decidió ingresar al mercado europeo, presentó los permisos y certificados que se le otorgaron en los Estados Unidos y consiguió la autorización para operar en Europa. Sin embargo, en Europa los funcionarios de ministerios no gozan del mismo prestigio y respeto que la FDA de los Estados Unidos. La opinión pública francesa rechazó el producto aduciendo que no era natural, que el pan no sería el mismo. También se opusieron los partidos políticos verdes, que son muy poderosos, las asociaciones de consumidores y las asociaciones de agricultores, que se negaban a comprar cada año las semillas. Finalmente, la posición de todos estos grupos ganó, se determinó que el producto no podía utilizarse y la empresa tuvo que retirarse. Al ver el ejemplo de los europeos, los agricultores de Estados Unidos se preguntaron por qué ellos no podían actuar de la misma manera. Resultado: Monsanto se fue a la quiebra y se vendió rápidamente a una empresa sueca para evitar perderlo todo. En la operación, la división de semillas y biotecnología de Monsanto, integrada por los mejores científicos del mundo, aproximadamente dos mil, fue valorizada en cero dólares. ¿Cuáles fueron las causas de este error? Faltó una estrategia completa de marketing que considerara no sólo el ámbito institucional, sino también a los consumidores y los grupos de influencia, lo que se enseña en el primer curso de marketing. Falló la cultura empresarial y faltó el acercamiento con el mercado y el entorno.
El error número seis consiste en sufrir de miopía competitiva en un mundo globalizado. En este caso me voy a referir a Marks \& Spencer, una empresa de confecciones inglesa conocida por sus diseños clásicos y tradicionales y que goza de gran fama por la extraordinaria calidad de sus productos, tanto así que los franceses y alemanes solían ir una vez al año a Londres para comprar trajes de esta marca.

La empresa se lanzó al mercado europeo con gran éxito, con los mismos productos que ofrecía al mercado interno. Como los ingleses son muy clásicos y tradicionalistas, cambiaron en nada o en muy poco sus modelos. Supusieron que bastaba que el mundo admirara a la reina Isabel, la princesa Diana o, ahora, a Camila. Pensaron que los únicos que podían competir con la moda inglesa eran los italianos y los franceses, pero, desafortunadamente para ellos, la competencia provino de Suecia y de España. ¿Quién podía pensar que los suecos y españoles podían incursionar en el negocio de confecciones? Después del desarrollo de las empresas suecas y españolas, Marks \& Spencer perdió una gran porción de participación en el mercado y, hace seis meses, dándose cuenta de la catástrofe económica, decidió cerrar 26 puntos de venta en toda Europa, con lo cual ha dejado sin empleo a más o menos 8 mil trabajadores. La responsabilidad en este caso corresponde, nuevamente, a la prepotencia empresarial y a la falla de los sistemas de información de la empresa.

El error número siete se presenta cuando la notoriedad no está relacionada con la satisfacción del consumidor. Es el caso de la empresa Benetton, que ustedes conocen muy bien, y que ilustra tanto el gran éxito como el gran fracaso. Logró el 
gran éxito cuando lanzó el eslogan «The United Color of Benetton» y expresó el concepto de que la mezcla de colores, cualesquiera que fueran, era correcta. La idea apelaba a la rebeldía de los jóvenes contra sus padres, que les enseñaban a no mezclar determinados colores. Bajo el concepto The United Color de Benetton y la imagen de un grupo de jóvenes de distintas razas, una rubia, una negrita, un asiático, la empresa generó un choque de rebeldía en los jóvenes hacia los esquemas tradicionales de sus padres y creó una moda que pegó de manera impresionante. ¿Pero qué hizo Benetton después? Para mantener la atención, generó choques culturales; de promover el concepto de rebeldía, con el cual los jóvenes se identificaban, pasó a promover la libertad sexual -no sé si ustedes vieron la publicidad con la serie de condones de todos los colores-. Después la empresa continuó desarrollando una serie de temas en su publicidad: el sida, la pena de muerte -esta vez presentando el rostro de condenados a muerte en los Estados Unidos-. Para entonces la gente ya no veía con simpatía la publicidad de Benetton, porque no podía identificarse con esos temas. La empresa buscaba la notoriedad solamente por la notoriedad en sí y terminó generando una corriente de opinión que no estaba de acuerdo con sus ideas. En los siete últimos años, Benetton tuvo que cerrar $30 \%$ de sus puntos de venta en el mundo entero, una proporción muy grande. El gran éxito causó el fracaso, porque la empresa no se dio cuenta de que hay necesidad de relacionar los mensajes con los conceptos de satisfacción y de satisfactor que se vende. Los responsables del error en este caso fueron el orgullo y la vanidad de los dirigentes y la incapacidad de entender los conceptos de satisfactor y satisfacción.
Ahora, al darse cuenta del problema, Benetton recorre el mundo tratando de demostrar que lo que hacía era arte, obedeciendo a la responsabilidad social de la empresa. Pero en la actualidad las tiendas de Benetton manejan otro concepto de publicidad en paralelo.

El error número ocho consiste en suponer que el mercado va a integrar lo obvio de inmediato, cuando en realidad el mercado no es naturalmente proactivo ni rápido en integrar lo obvio. Vamos a tomar el caso de un banco, el BBL (Banque Bruxeles Lambert), que es el número dos en Bélgica. Seis años atrás, este banco decidió que, dado que se formaban colas en las agencias, lo obvio era establecer que todas las transacciones pudieran hacerse por teléfono, computadora o cajero automático, de modo que las agencias se dedicaran solamente a atender a empresas y a brindar asesoría, pero ya no a atender transacciones corrientes. Se pensó que, naturalmente, era más fácil y menos costoso utilizar el teléfono y dar el número de cuenta que tener que desplazarse hasta una agencia bancaria.

¿Qué sucedió en realidad? Cuando se implementa una estrategia como ésta, los empleados piensan que van a perder su puesto. Luego, el primer problema fue que los empleados empezaron a hacer comentarios negativos sobre el banco, algo así como: «Este banco no sirve para nada, no le importan sus clientes, no le importa la relación humana». El segundo problema surgió de parte de los clientes, generalmente personas de edad madura para quienes ir a la agencia bancaria es parte de una dimensión social, dado que les da la posibilidad de salir y conversar con alguien más que con la empleada de la casa y el televisor. Si no tienen excusa 
para salir a la calle, el día para estas personas se vuelve muy aburrido. ¿Resultado? El banco perdió participación en el mercado y, con inestabilidad interna altísima, fue comprado hace tres años por empresarios holandeses. Otro error de marketing muy grande y en el cual la responsabilidad corresponde a la falta de análisis de la dinámica del mercado, a una gerencia demasiado alejada de las expectativas y percepciones de sus clientes.

El error número nueve ocurre cuando la búsqueda de nuevos productos para aumentar el volumen de ventas hace perder el norte de la empresa. Es el caso, muy interesante, de la empresa GB. Adaptando el concepto americano de supermercado, los directivos de la empresa empezaron a lanzar supermercados en Europa, con un éxito tan grande que vendían el $30 \%$ de todos los productos alimenticios de un país, una proporción enorme. Los directivos de la empresa se dieron cuenta, entonces, de que los clientes necesitaban más servicios de los que los supermercados les ofrecían, y agregaron grandes almacenes al negocio. Después advirtieron que las amas de casa necesitaban talleres que fabricaran llaves y también talleres de reparación de zapatos, y decidieron establecer pequeños talleres para llaves y zapatos afuera de los supermercados. Luego observaron que algunas personas preferían supermercados muchos más chicos cerca del hogar, e ingresaron a este negocio. Había también la posibilidad de establecer supermercados de 10 mil ó 15 metros cuadrados, los supergrandes, y empezaron con un surtido más grande. Pensaron además que los clientes necesitaban algo de comer, y se lanzaron al negocio de los fast food con las hamburguesas Quick. Después se dieron cuenta de que para todas esas cosas se necesi- taba transporte y de que si ellos podían ofrecer este servicio obtendrían más utilidades, y se lanzaron al negocio de transporte. Luego, como varios de sus negocios insumían carne, ingresaron también al rubro de carnicería. Y como donde hay amas de casa hay niños, incursionaron también en el ramo de juguetería, y luego en perfumes y en artículos para deportes. Al final incursionaron en tantos rubros que el negocio no podía manejarse eficientemente y sobrevivía no con las utilidades, sino con su poder de negociación con los proveedores. De ser empresa productiva, GB se convirtió en empresa financiera. Luego, a raíz de los problemas surgidos al interior de la Unión Europea acerca de la relación entre los países grandes y pequeños, la empresa empezó a enfrentar dificultades hasta que hace tres meses desapareció; fue comprada por la empresa francesa Carrefour. La nueva propietaria ha limpiado el negocio y vuelto al concepto de base. Agregar negocios marginales que hacen perder el norte a la empresa es consecuencia de un concepto muy cortoplacista del marketing, de una orientación hacia la «venta» y de una visión muy limitada; en suma, los responsables del error gerencial.

El error diez ocurre cuando el éxito de una función o árrea de la empresa pone en peligro todo el resultado de la empresa. Al respecto hay dos casos interesantes, el de Quantum y el de Midland. Quantum era una empresa de confecciones que incursionó en el comercio electrónico. Contaba con un magnífico catálogo de productos y, al mismo tiempo, con una extraordinaria capacidad de marketing y de publicidad, al punto que obtuvo un éxito notable. Pero los directivos no repararon en los aspectos administrativos y logísticos, no pensaron que los 
problemas se originarían por fallas en la atención de los pedidos. Cuando los clientes realizan una compra a través del comercio electrónico, esperan recibir su producto de inmediato, no en dos meses. ¿Resultado? La gente abandonó Quantum y la empresa tuvo que cerrar. Las pérdidas sumaron millones de dólares y dieron origen a la más grande quiebra en el comercio electrónico desde el desarrollo de la nueva tecnología. Otro caso que alcanzó gran resonancia fue el de Midland, empresa supuestamente muy bien manejada que al mismo tiempo que anunciaba en la bolsa de París las más grandes utilidades de todos los tiempos, anunciaba también el cierre de cinco plantas en Europa. ¿Cómo fue posible esto? Faltó comunicación interna, falló la relación entre las funciones de marketing, finanzas y producción. ¿ Resultado? Midland está perdiendo participación en el mercado. Caso típico cuando una función cree que su punto de vista es el único que cuenta para manejar la empresa. ¿Los responsables? Las fallas en el sistema de toma de decisiones y en la coordinación, comunicación y recursos.

El error once consiste en la falta de convencimiento y ambición que conduce al fracaso. Éste es el caso de la cerveza Hoegaarden, cerveza blanca -llamada así porque no se ve a través de ella- lanzada al mercado por una sola persona hace más o menos 25 años, con gran éxito. Empezó vendiendo $25 \mathrm{mil}$ hectolitros al año y ocho años después vendía $300 \mathrm{mil}$ hectolitros de cerveza. La empresa Interbrew, viendo que esta pequeña empresa le estaba quitando mercado, le propuso a su propietario, el señor Celis, la compra de la planta por una suma muy atractiva. Puesto que hay ofertas que no se pueden rechazar, el propietario aceptó y mientras él disfrutaba de vacaciones, Interbrew incrementó sostenidamente la producción. Al cabo de cinco años vendía aproximadamente dos millones de hectolitros de cerveza. Con estos resultados, Interbrew intentó ingresar al mercado de Estados Unidos, pero no lo logró y, más bien, perdió una considerable suma. Como no es una cerveza transparente, los consumidores de ese país la rechazaron porque les parecía poco limpia. Interbrew mantuvo la cerveza en Europa, donde se vende bien, pero ya no con la gran tasa de crecimiento de años anteriores. Mientras tanto, Celis dio fin a sus vacaciones y lanzó una nueva cerveza, CelisWhite, en Texas. Hoy en día CelisWhite es la cerveza americana que crece a mayor ritmo, al punto que está siendo importada por Bélgica. Ésta es la historia de David y Goliat. Un empresario pequeño que cree en su producto frente a una gran empresa para la cual ese producto es solamente uno más. ¿Los responsables del error? La falla del sistema de información y la falla de visión de la alta gerencia.

El error doce ocurre cuando el entorno genera una autolimitación emprendedora. ¿Qué significa esto? Sin darse cuenta las empresas dejan de innovar, de diseñar nuevos productos, no buscan conquistar nuevos mercados, es decir, se autocensuran. ¿Por qué? Porque el entorno no es alentador, el mercado no responde o porque hay incertidumbre frente a las medidas del gobierno. Un caso interesante al respecto es, nuevamente, el de Interbrew. Esta empresa tiene una posición dominante en varios países europeos y por esta posición dominante la cerveza se vende. El satisfactor es el placer, y uno obtiene placer cuando acude a un bar agradable. Si no se es alcohólico o se está borracho, la cerveza como tal no interesa 
mucho, lo que es más importante es el contexto social en el que se consume. Interbrew tenía como dimensión principal bares, cafés, restaurantes y, en general, establecimientos que generaban mucha venta de cerveza. Frente a este hecho, la Unión Europea la acusó de tener una posición dominante, porque comprando los establecimientos no daba a las otras cervezas la posibilidad de vender. Interbrew separó el negocio de los establecimientos del negocio de la cerveza. Igualmente, no quiso ingresar al negocio de microcervecerías porque sus directivos pensaron que de nuevo la Unión Europea los iba a acusar de monopolistas. ¿Resultado? Abrieron la puerta a otros competidores. Actualmente, el mercado de la microcervecería es el de mayor crecimiento en toda Europa y en los Estados Unidos. Por autolimitación, Interbrew no ingresó a este mercado y dejó crecer a otros. ¿Responsables? La cultura empresarial y organizacional.

El error número trece se presenta cuando la dimensión técnica oculta la dimensión estratégica. Eso ocurre en muchas empresas, especialmente en el campo de la publicidad porque el publicista con su técnica y sus efectos impresiona al resto de la empresa. Voy a tomar el caso del Milenium Dome. El Milenium Dome era un gran logro técnico, no sólo por la arquitectura, sino por una serie de posibilidades y atracciones, tipo Steven Spielberg, que ofrecía. Una de ellas, por ejemplo, era un cuerpo humano por cuyas arterias y corazón se podía caminar. Todo ello atrajo a mucha gente, pero en realidad, comparado con Disneylandia y otras ciudades del futuro, no era tan extraordinario. ¿El resultado? Cuando se mide solamente el aspecto técnico y se oculta el aspecto estratégico, en la mayoría de los casos se genera el fracaso. ¿Responsa- ble? La incompetencia de la gerencia de marketing.

¿Qué lecciones podemos sacar de todo esto? ¿Cuáles son las mayores fuentes de errores? En primer lugar, la falta o falla en el análisis del mercado y de su entorno, base principal de cualquier curso de gerencia. En segundo lugar, la cultura empresarial y organizacional: falta de visión, toma de decisiones sin consenso, comunicación insuficiente, problemas de recursos humanos y de incapacidad para asumir la incertidumbre. Este último aspecto es muy importante. La capacitación profesional no pone énfasis suficiente en la necesidad de aprender a manejar la incertidumbre. Estamos en un mundo lleno de incertidumbre, tenemos que aprender a vivir con ella y, sin embargo, lo único que se enseña es a promover la certidumbre. Más importante que hacer grandes planes es la capacidad de generar flexibilidad dentro de una organización para adaptarse a cualquier ruptura del entorno. En tercer lugar, la falta de un adecuado sistema de información. Hoy existe la tecnología para tener un buen sistema de información. En el caso del marketing, el CMR es una base que no existía diez años atrás. En cuarto lugar, la incapacidad o incompetencia de los gerentes para dominar lo técnico o tecnológico. Aquí está incluido también el problema de gerentes que no se reactualizan y no entienden la técnica que utilizan. En quinto lugar, el hecho de que los canales de comunicación o de información no funcionan o están cortados. Un error clásico que se comete con frecuencia es ya no hablar con los vendedores ni con los clientes cuando uno se convierte en gerente.

¿A qué conclusiones se puede llegar? El concepto de marketing, más que nunca, es válido y valioso, pero se tiene que 
volver a la base, al sentido común. El sentido común es el elemento más importante al que debe recurrirse antes de aplicar de manera ciega las herramientas. ¿Y qué se debe hacer? Primero, diseñar la misión de la empresa dentro de su entorno y hacer que todos la compartan, y compartan también los objetivos correspondientes. Segundo, elaborar el «satisfactor» que generará el «capital de lealtad y satisfacción en el mercado». Es decir, un capital que se debe mantener y evolucionar a través del tiempo. Tercero, asegurarse del buen desempeño de la función estratégica (con sus objetivos, su sistema de información y el comportamiento gerenial) y de la capacidad organizacional de la empresa (acercamiento interfuncional), porque eso facilitará la implementación. Y finalmente, prestar atención al hombre, que es el recurso más importante, porque si no hay hombres con visión, si no se mantiene capacitado al recurso humano, abierto a nuevas opciones que le permitan tomar distancia del día a día, el fracaso puede estar muy cerca. 